\title{
Hybrid multispectral optoacoustic and ultrasound tomography for morphological and physiological brain imaging
}

Ivan Olefir

Elena Merčep

Neal C. Burton

Saak V. Ovsepian

Vasilis Ntziachristos 


\title{
Hybrid multispectral optoacoustic and ultrasound tomography for morphological and physiological brain imaging
}

\author{
Ivan Olefir, ${ }^{\mathrm{a}, \mathrm{b}}$ Elena Merčep, ${ }^{\mathrm{a}, \mathrm{c}, \mathrm{d}}$ Neal C. Burton, ${ }^{\mathrm{d}}$ Saak V. Ovsepian, ${ }^{\mathrm{a}, \mathrm{b}}$ and Vasilis Ntziachristos ${ }^{\mathrm{a}, \mathrm{b}, *}$ \\ ${ }^{a}$ Helmholtz Zentrum München, Institute for Biological and Medical Imaging, Ingolstädter Landstraße 1, Neuherberg 85764, Germany \\ ${ }^{\text {b}}$ Technische Universität München, School of Bioengineering, Boltzmannstraße 11, Garching 85748, Germany \\ ${ }^{\circ}$ Technische Universität München, Faculty of Medicine, Ismaninger Straße 22, Munich 81675, Germany \\ diThera Medical GmbH, Zielstattstrasse 13, Munich 81379, Germany
}

\begin{abstract}
Expanding usage of small animal models in biomedical research necessitates development of technologies for structural, functional, or molecular imaging that can be readily integrated in the biological laboratory. Herein, we consider dual multispectral optoacoustic $(\mathrm{OA})$ and ultrasound tomography based on curved ultrasound detector arrays and describe the performance achieved for hybrid morphological and physiological brain imaging of mice in vivo. We showcase coregistered hemodynamic parameters resolved by OA tomography under baseline conditions and during alterations of blood oxygen saturation. As an internal reference, we provide imaging of abdominal organs. We illustrate the performance advantages of hybrid curved detector ultrasound and $\mathrm{OA}$ tomography and discuss immediate and long-term implications of our findings in the context of animal and human studies. @ 2016 Society of Photo-Optical Instrumentation Engineers (SPIE) [DOI: 10.1117/1.JBO.21.8.086005]
\end{abstract}

Keywords: hybrid systems; photoacoustic; brain tomography; oxygen saturation; multispectral optoacoustic tomography; in vivo imaging.

Paper 160245R received Apr. 14, 2016; accepted for publication Jul. 25, 2016; published online Aug. 12, 2016.

\section{Introduction}

Biomedical imaging has a profound impact on interrogation of the structure and functional dynamics of living organisms, with major implications for diagnostics and healthcare. ${ }^{1,2}$ Combined with advances in molecular biology and genetics that spurred the development of small animal models, in vivo imaging is increasingly considered for a range of basic and translational research applications. Amid imaging modalities implemented for clinical and basic investigations, ultrasonography (US), magnetic resonance imaging, single-photon emission, positron emission, or X-ray computed tomography (PET and XCT), have been most widely considered for small animal studies. ${ }^{3-8}$ In addition, optical methods, such as optical coherence tomography or multiphoton microscopy, have been used in vivo at cellular and subcellular resolution, ${ }^{9,10}$ but at imaging depths limited to a few millimeters. ${ }^{11}$

Recently, small animal optoacoustic (OA) imaging and, in particular, multispectral optoacoustic tomography (MSOT), has been employed to bridge the gap between the optical microscopy and radiological methods. ${ }^{11-14}$ The technique is based on the thermoelastic expansion of the tissue after absorption of transient light energy, with latter giving rise to ultrasonic waves. ${ }^{15}$ Image formation is based on detecting generated waves and application of mathematical inversion to determine the optical contrast (absorption) acting as the source of sound. Typically, laser pulses in the 10- to 100-ns range are implemented to induce an ultrasonic response, although continuous-wave intensity-modulated lasers have also been shown to

*Address all correspondence to: Vasilis Ntziachristos, E-mail: v.natziachristos@ tum.de produce acoustic signals. ${ }^{12,16,17}$ The major advantage of MSOT over conventional optical imaging methods is that the image quality is less prone to distortion by photon scattering within the specimen. Therefore high-resolution images of the deep structures can be produced with ultrasound diffraction-limited resolution. ${ }^{11,18}$ By illuminating at multiple wavelengths and using spectral unmixing techniques, MSOT can resolve the contributions of different endo- and exogenous photoabsorbing molecules, nanoparticles, or other labels, based on their absorption. ${ }^{19-21}$ This spectral capacity of MSOT affords its molecular specificity, capable of distinguishing various endogenous contrasts, including oxy- and deoxy-hemoglobin, melanin, and others, or changes in their functional states. For this reason, MSOT is customarily considered in studies of physiological and pathophysiological processes as well as for molecular imaging. ${ }^{22-24}$ A large number of extrinsically administered contrast-enhancing agents is also widely utilized for expanding its applications toward addressing a range of biological questions and unmet clinical need ${ }^{16,18}$ at depths and resolutions not feasible with conventional imaging approaches. ${ }^{25-28}$

It has been nevertheless recognized that the combination of OA imaging with other imaging modalities can enhance the MSOT capabilities and applications. This principle was applied in hybrid microscopic studies, where the merger of two-photon and OA microscopy in a single system affords high-resolution imaging of fluorescence, second-harmonic, and optical absorption contrasts within the same specimen, enriching the features resolved. ${ }^{29}$ The combination of OA macroscopy with ultrasound imaging has also been considered, taking advantage of a shared signal detector. A commonly used

$1083-3668 / 2016 / \$ 25.00$ @ 2016 SPIE 
approach implements linear US arrays with integrated fiberbased light delivery systems, where OA and ultrasound data are acquired in a sequential fashion. ${ }^{30,31}$ Due to its wide availability and use in the clinical US systems, the linear arrays have been previously adopted for hybrid OA and US imaging, through modification of the existing probes. Such a design therefore inherits flexibility of US modality in terms of access to various measurement sites. However, it has been shown that linear ultrasound detectors do not offer optimal OA image quality. ${ }^{32,33}$ Conceivably, the use of concave ultrasound detectors greatly improves the quality and accuracy of images, due to improved angular coverage of the specimen under investigation. ${ }^{34}$ This however involves the use of ring-shaped detectors arrays, which limits the flexibility of such hybrid systems and their use to human studies, but with preserved applications for small animal imaging. A hybrid OA-US system with ring detector geometry was proposed by Xia et al., ${ }^{35}$ which used laser illumination of the ring array to generate US pulses. With such approach, the speed of sound within the field of view has been estimated to enhance the quality of OA images. It should be noted, however, that in this study, US played a supporting role in OA image reconstruction and did not yield high-resolution structural images. We have recently introduced a hybrid imaging system consolidating a concave detection array-based ultrasound tomography (UST) with MSOT, using the same array detector, capable of concurrently obtaining OA and ultrasound readouts. ${ }^{36}$ The system was shown to generate hybrid images of the bladder, using US and near-infrared dye perfused in the bladder in vivo. Nevertheless, the ability of such a system to generate coregistered physiological (hemodynamic) and structural data has not been fully explored.

In this study, we implemented hybrid MSOT-UST imaging for correlative morphological and functional measurements of the mouse brain. We evaluate anatomical UST data with physiological information of MSOT and compare the performance of MSOT with 256 and 512 detector elements. As a reference, we investigate the performance of the hybrid system for imaging of abdominal organs. Finally, we project onto future applications and potential challenges related with use of dual MSOT-UST imaging for basic and translational physiology and neuroscience research and discuss avenues for further improvements.

\section{Materials and Methods}

\subsection{Hybrid Ultrasound and Optoacoustic Tomography}

Figure 1 presents a schematic of the hybrid imaging platform with main components. The principal module shared between both systems is a 512-element concave ultrasound detector array (DA) [Imasonics SaS, Voray, France; array type: spherical concave array covering $270 \mathrm{deg}$; number of channels: 512 ; mechanical focalization: toroidal focusing; radius of curvature SR $40 \mathrm{~mm} \pm 1 \mathrm{~mm}$; radius of the elements (elevation): $37 \mathrm{~mm}+$ $1 /-2 \mathrm{~mm}$; elementary pitch $(p): \sim 0.735 \mathrm{~mm} / \sim 0.37 \mathrm{~mm}$; interelement spacing $(e): 0.1 \mathrm{~mm}$; width of the elements $(h)$ : $15 \mathrm{~mm}$ (chord); center frequency ( $-6 \mathrm{~dB}): 5 \mathrm{MHz} \pm 10 \%$; bandwidth $(-6 \mathrm{~dB}): \geq 55 \%$ in transmit/receive mode] coupled to laser beam and controlling multiplexer (MUX). The concave multielement detector is connected to two data acquisition (DAQ) systems, each corresponding to one of the acquisition modes. Despite a common mechanism of US and OA signal detection, a second DAQ has to be employed due to (1) conventional OA DAQ electronics not supporting the transmission of US pulses and (2) differences in amplification and input impedance needed to acquire readouts from the two modalities. The data are stored and processed on a personal computer (PC). In the multispectral tomography mode [MSOT, Fig. 1(a)], short laser pulses pass through a diffuser, to ensure even illumination of the specimen. The ultrasound waves produced due to the thermoelastic expansion of the specimen are detected and processed to form images with proprietary software (iThera Medical $\mathrm{GmbH}$, (a)

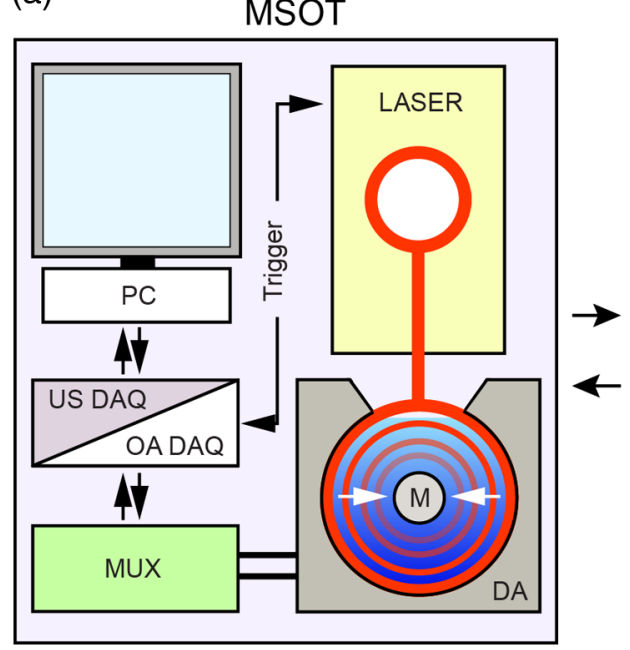

(b)

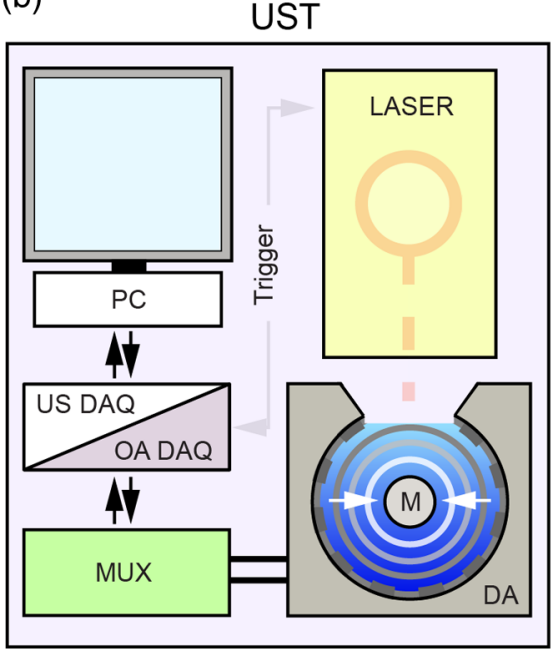

Fig. 1 Hybrid MSOT-UST imaging system: a schematic. (a) MSOT: a laser pulse triggers the DAQ system and illuminates the mouse $(\mathrm{M})$ generating the ultrasonic waves due to light absorption, which are sampled by transducers on a detector ring and reconstructed into an image by PC. (b) UST: the mouse is interrogated by US waves that are generated by the transducers on the DA; the US waves reflected from within the sample due to the acoustic impedance mismatch of the different tissues are subsequently detected and processed into an image. MUX is been used for switching between various acquisitions modes. 
Munich, Germany). In the UST acquisition mode [Fig. 1(b)], the specimen is interrogated by US pulses through an array and reflected signals are sampled with acoustic sensors as specified elsewhere. $^{36}$

A holder consisting of a semiflexible light- and soundpermeable polyethylene membrane is used to horizontally mount the animal into the center of the concave DA. For optimal sound coupling, the detector and the animal holder are immersed in water. The plastic membrane separates the animal from being in contact with the water, while offering $~ 360$ deg of water coupling and $\sim 90 \mathrm{deg}$ of open access to the animal from the top. In both MSOT and US acquisition modes, one two-dimensional (2-D) cross-sectional plane ( 1-mm thick) is scanned at a time. Plane sectioning is performed by horizontal translation of the animal holder with respect to the detection ring with a minimum step of $0.1 \mathrm{~mm}$. Due to the unconventional geometry of the detector used, specific reconstruction algorithms have to be employed to form a US image. ${ }^{37}$ To switch from one acquisition mode into another, a custom made multiplex programmable switchboard was used. ${ }^{36}$ For MSOT, 9-ns laser pulses of different wavelengths have been used $(\lambda=715,730,775,800,850$, and $900 \mathrm{~nm}$ ), which were produced with an Nd:YAG-pumped optico-parametric modulator (InnoLas Laser GmbH, Krailling, Germany) at $10 \mathrm{~Hz}$ and per-pulse energy of $120 \mathrm{~mJ}$, with the intensity kept constant throughout the imaging sessions (laser pulse fluence on the surface of the imaged objects under $20 \mathrm{~mJ} / \mathrm{cm}^{2}$ for similar illumination ${ }^{38}$ ). Affirmatory imaging of used specimen at specified above wavelengths was also performed using a commercial MSOT scanner (MSOT256-TF, iThera Medical GmbH, Munich, Germany), with OA signals collected with a 256-element detector ring. ${ }^{34}$ MSOT images have been acquired at $10 \mathrm{~Hz}$ and averaged 10 frames per wavelength, to reduce the variability due to the breathing motion and improve the signal-to-noise ratio.

\subsection{Animals and Experimental Procedures}

Young adult mice (Hsd:Athymic Nude-Foxn1nu/nu) were used for these experiments, which were housed in the animal housing facility $\left(21^{\circ} \mathrm{C} \pm 2{ }^{\circ} \mathrm{C}\right.$, humidity $36 \% \pm 2 \%$ at $12 / 12$-h light/dark cycle) at the Institute of Biomedical Imaging, Helmholtz Zentrum München, with food and water provided ad libitum. All procedures involving animal experimentations were conducted according to the institutional guidelines and the government of Upper Bavaria and complied with German Federal and EU law. Efforts were made to reduce the animal usage and suffering.

MSOT and UST of the head and abdominal organs were conducted and related to anatomical references. ${ }^{39}$ Experiments were carried out under general anesthesia (1.8\% isoflurane in $100 \%$ $\mathrm{O}_{2}$ at $0.81 \mathrm{ml} / \mathrm{min}$ ). Baseline measurements at normal breathing were followed by a gas challenge. In total, four breathing states were cycled with supplied gas composition altered as follows: (1) medical air, (2) a mixture of medical air and $10 \% \mathrm{CO}_{2}$, (3) $100 \%$ oxygen, and (4) medical air. Every breathing condition lasted for $\sim 2 \mathrm{~min}$ resultant in a total of $\sim 8 \mathrm{~min}$ per imaging session.

\subsection{Data Analysis and Presentation}

Raw data have been analyzed off-line in MATLAB ${ }^{\circledR}$. Signals were filtered with a bandpass filter $(400 \mathrm{kHz}$ to $8 \mathrm{MHz}$ ) with multispectral images reconstructed using a model-based algorithm ${ }^{40}$ resulting in 2-D images of the measured planes. MSOT images of the head region taken at $900 \mathrm{~nm}$ with MSOT512 and MSOT256 were automatically coregistered in MATLAB ${ }^{\circledR}$. For resolution comparison, the intensity of selected high-contrast regions of interest (ROIs) have been profiled; data were extracted from corresponding locations of coregistered images using MATLAB $^{\circledR}$ and plotted in arbitrary units (a.u.). Intensity profile graphs were fitted using Gaussian function with full width at half maximum (FWHM) taken as an indication of the resolving power and compared between two (MSOT256 and MSOT512) systems. For correlation of MSOT and UST readouts, semiautomatic data registration was performed in MATLAB $^{\circledR}$ by matching three pairs of control points selected manually at the corresponding anatomical landmarks defined on both MSOT and US images. Anatomical MSOT images were corrected for light fluence computed with a finite element solution to diffusion approximation assuming homogeneous absorption of $0.3 \mathrm{~cm}^{-1}$ and reduced scattering of $10 \mathrm{~cm}^{-1}$. For studies of blood oxygenation dynamics, measurements were made with MSOT while inhaled gas content was altered as specified above. Prior to data unmixing, the MSOT readouts were calibrated to account for the absorption by a 4-cm-thick layer of water. The ROIs of functional recordings, which included blood vessels and parenchymal tissue distal from major blood supply routes, were defined for tracking the oxygenation saturation $\left(\mathrm{sO}_{2}\right)$ changes overtime. Calibrated multispectral data were subsequently unmixed based on absorption spectra of oxy- and deoxy-hemoglobin using a linear curvefitting algorithm $\left(\right.$ MATLAB $\left.^{\circledR}\right)$. No optical fluence correction was used prior to unmixing. The values for $\mathrm{sO}_{2}$ were averaged over respective ROIs, with oxygenation levels computed relative to the baseline (denoted as "relative $\mathrm{sO}_{2}$ " in tables and figures) defined as the average oxygenation level in the kidney area prior to the breathing challenge. The resulting temporal profiles from the ROIs in the abdominal area were processed with median filter of order 7 to mitigate the noise. To characterize the dynamic response of tissue to gas challenge, data corresponding to the timeframes of 220 to $290 \mathrm{~s}$ for brain ROIs and 220 to $355 \mathrm{~s}$ for abdominal ROIs were extracted from the selected profiles, with values normalized to the range from 0 to 1 for individual profiles. Sigmoid functions of $1 /\left(1+e^{\tau(x-b)}\right)$ were fitted to the extracted data, where $x$ denotes time, $b$ characterizes the center of the curve, and $\tau$ governs steepness of ascent (higher values mean faster rise). Obtained values of $\tau$ are summarized in Table 1. Graphs and color maps have been generated in MATLAB ${ }^{\circledR}$ or in EXCEL; the final figures were prepared using IgorPro (6.1) and Adobe Illustrator (CS6).

\section{Results}

To validate the performance of the hybrid system and its utility for correlative UST and MSOT in vivo, we imaged the brain and abdominal organs of living mice under normal conditions and during alterations of $\mathrm{sO}_{2}$ induced by gas challenge.

\subsection{Hybrid Imaging of the Brain}

Figure 2 shows a cryosection of the mouse head ex vivo annotated for major anatomical references of the brain (a), with corresponding UST and MSOT cross sections obtained in vivo (b and c, respectively). The MSOT image is labeled to highlight the regions chosen for the analysis of hemodynamics. As evident, high-contrast structures with distinct contours can be 
Table 1 Summary of $\mathrm{sO}_{2}$ dynamics and corresponding values. Anatomical references are numerated in accordance to the MSOT images in Figs. 2 and 4.

\begin{tabular}{|c|c|c|c|c|c|}
\hline & Organ & Anatomical references & Baseline $\mathrm{sO}_{2}$ (Rel. \%) & Peak $\mathrm{sO}_{2}($ Rel. \%) & $\mathrm{sO}_{2}$ rise $(\mathrm{tau})$ \\
\hline \multirow[t]{12}{*}{ Vessels } & \multirow[t]{6}{*}{ Head } & Sagittal sinus (1) & 118 & 178 & 0.607 \\
\hline & & Maxillary artery (2) & 128 & 194 & 0.329 \\
\hline & & Medial cerebral artery (5) & 131 & 176 & 0.519 \\
\hline & & Maxillary artery (6) & 123 & 185 & 0.293 \\
\hline & & Mean & 125 & 183 & 0.437 \\
\hline & & Std & 6 & 8 & 0.151 \\
\hline & \multirow[t]{6}{*}{ Abdominal } & Sup. vessel (3) & 125 & 155 & 0.090 \\
\hline & & Sup. vessel (2) & 135 & 167 & 0.061 \\
\hline & & Sup. vessel (6) & 121 & 152 & 0.068 \\
\hline & & Spinal br. of Lumb. art (4) & 134 & 173 & 0.231 \\
\hline & & Mean & 129 & 161 & 0.112 \\
\hline & & Std & 7 & 10 & 0.081 \\
\hline \multirow[t]{8}{*}{ Parenchyma } & \multirow[t]{4}{*}{ Head } & Brain (3) & 116 & 150 & 1.302 \\
\hline & & Brain (4) & 102 & 144 & 1.264 \\
\hline & & Mean & 109 & 147 & 1.283 \\
\hline & & Std & 10 & 4 & 0.027 \\
\hline & \multirow[t]{4}{*}{ Abdominal } & Kidney (1) & 100 & 113 & 0.065 \\
\hline & & Kidney (5) & 110 & 131 & 0.099 \\
\hline & & Mean & 105 & 122 & 0.082 \\
\hline & & Std & 7 & 13 & 0.024 \\
\hline
\end{tabular}

distinguished in both UST and MSOT images, although with notable differences. In MSOT cross sections, the heterogeneous optical absorption at various depths unveils discrete outlines of major vascular elements such as the dorsal sagittal sinus, medial cerebral, maxillary and internal carotid arteries along with the contours of the parietal cortex, hippocampal formation, and optic chiasm. ${ }^{39}$ On corresponding UST images, strong contrasts due to ultrasound impedance mismatch highlight the skin to skull interface, with most of the signal provided by thick cranial bones causing strong reflection of the US waves while the brain itself appears obscured. As evident from the overlaid UST and MSOT tomograms [Fig. 2(d)], the combination of two modalities results in coregistered image acquired with the shared ultrasonic DA.

Due to its spectral dimension, ${ }^{18}$ MSOT can obtain readouts of the brain hemodynamics and $\mathrm{sO}_{2}$ in conjunction with anatomical data in vivo. Figures 2(e) and 2(f) show images of the dorsal compartments of mouse brain overlaid with colorcoded oxygenation maps corresponding to different breathing conditions (air and $100 \% \mathrm{O}_{2}$, respectively) with supply of $100 \% \mathrm{O}_{2}$ increasing tissue oxygenation levels. The overall dynamics of the $\mathrm{sO}_{2}$ in defined ROIs [Figs. 2(e), 2(f); enboxed 1 and 4 ] of the dorsal cerebrum with their changes related to the breathing conditions are summarized in Figs. 2(g) and 2(h), respectively (blue circles and error bars represent correspondingly mean relative oxygenation and standard deviation within respective ROIs). After a stable baseline recording, supply of $10 \% \mathrm{CO}_{2}$ causes deoxygenation of the brain tissue and sagittal sinus while supply of $100 \% \mathrm{O}_{2}$ leads to a steep increase in $\mathrm{sO}_{2}$ in both areas.

To evaluate the kinetics of the hemoglobin gradient changes, we analyzed the rates of the relative $\mathrm{sO}_{2}$ changes upon $\mathrm{O}_{2}$ challenge. Figure 3 presents the graphs of the kinetics of $\mathrm{sO}_{2}$ in brain tissue (a) and sagittal sinus (b). Black circles denote normalized data points pulled from regions marked by arrows 3 (a) and 1 (b) in Fig. 2(c), while red lines correspond to the fitted sigmoid functions. Faster $\mathrm{sO}_{2}$ rates within the brain tissue are evident as compared to that of sinus, consistent with measurements of the $\mathrm{sO}_{2}$ dynamics and comparison between several brain areas and major blood vessels (Table 1). These findings suggest a tighter coupling of the oxygenation of the neural tissue with the breathing state. It is important to note that changes in $\mathrm{sO}_{2}$ induced by the gas challenge were reversible, confirming that the effects of experimental interventions were transient and remain within physiological limits, and were not associated with irreversible impairments of the brain hemodynamics. 
(a)

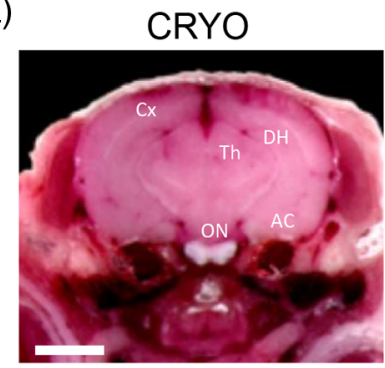

(c)

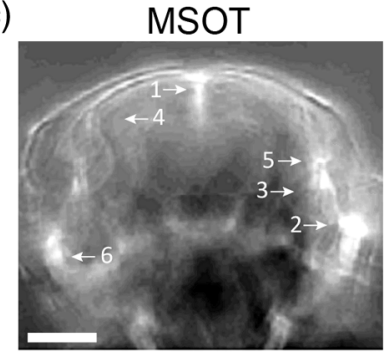

(b)

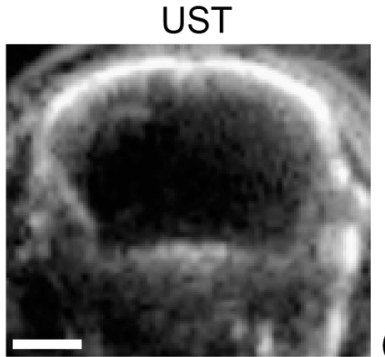

(d)

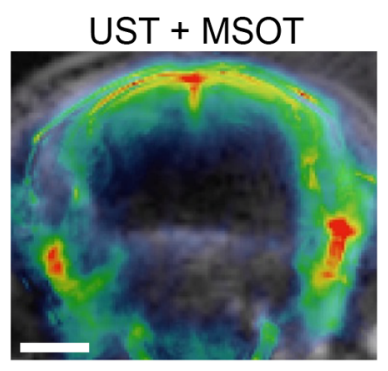

(e)

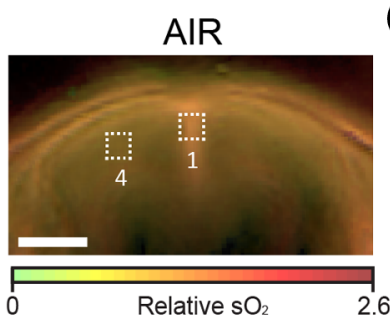

(f)

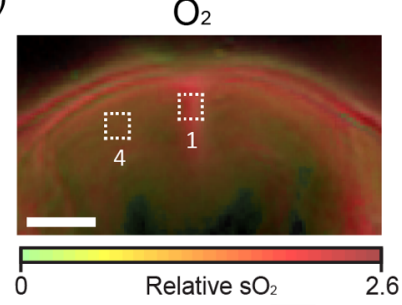

(g)

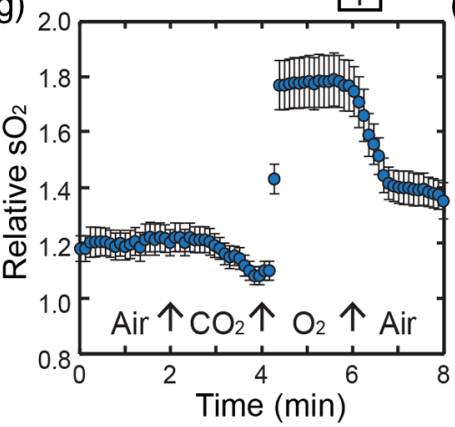

(h)

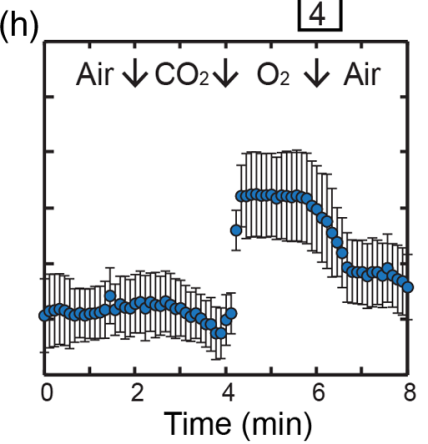

Fig. 2 Hybrid UST and MSOT imaging of the brain. (a) Annotated cross section of a mouse head (brain, coronal plane) with corresponding UST, MSOT, and overlaid images (b, c, and d, respectively). Scale bars: $2 \mathrm{~mm}$. Annotations: Cx, cortex; DH, dorsal hippocampus; Th, thalamus; AC, amygdalar complex; and $\mathrm{ON}$, optic nerve. The regions of interest (c, 1 to 6 ) for analysis of the $\mathrm{sO}_{2}$ dynamics are highlighted on MSOT image with arrows (with measurements summarized in Table 1). 1, sagittal sinus; 2 and 6, maxillary arteries; 3 and 4, parenchymal tissue; and 5, medial cerebral artery. (e and f) Color-coded relative $\mathrm{sO}_{2}$ maps obtained at indicated breathing phases overlaid with anatomical MSOT images. ( $\mathrm{g}$ and $\mathrm{h}$ ) Time courses of changes from respective regions of interests (enboxed, 1 and 4) corresponding to sagittal sinus $(\mathrm{g})$ and parietal cortex $(\mathrm{h})$. Blue circles and error bars denote mean relative $\mathrm{sO}_{2}$ and standard deviations of measurements within respective ROls, correspondingly. Experimental conditions are indicated within individual graphs. Scale bars: $1.8 \mathrm{~mm}$.

\subsection{Comparison of 256 Versus 512 Multispectral Optoacoustic Tomography}

To investigate if an increase in the number of ultrasonic detectors in hybrid MSOT-UST platform affects the image quality and resolution of the system, we compared the intensity profiles of defined ROIs on head cross-sectional images acquired with hybrid MSOT512 with those obtained by standalone MSOT256.

Figures 4(a) and 4(b) present an annotated histological cross section of a mouse head ex vivo (coronal section) taken at the plane that corresponds to $-5.3-\mathrm{mm}$ Bregma of the mouse brain atlas. ${ }^{41}$ Figures $4(\mathrm{c})-4(\mathrm{f})$ show tomographic cross sections of

(a)

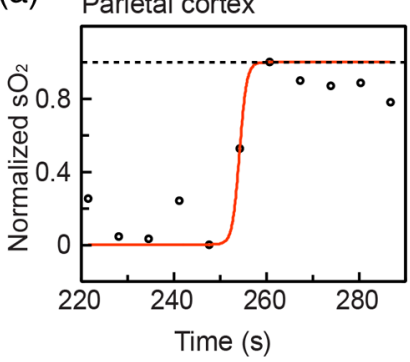

(b) Sagittal sinus

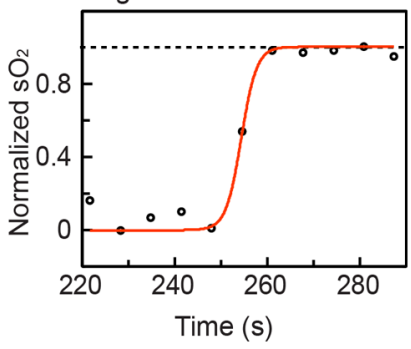

Fig. 3 Graphical representation of the kinetics of the $\mathrm{sO}_{2}$ within large blood vessels and parenchyma of the brain measured with MSOT. ( $a$ and b) Representative $\mathrm{sO}_{2}$ dynamics overtime pulled from ROls [corresponding to arrows: 3 and 1, in Fig. 2(c), respectively]. Individual data points overtime are fitted with sigmoid functions with values (tau) defining the slope summarized in Table 1. the same anatomical plane obtained with the MSOT512 (c and d) and MSOT256 (e and f) systems at 715-nm (c and e) and $900-\mathrm{nm}$ ( $\mathrm{d}$ and $\mathrm{f}$ ) wavelengths. As optical absorption of brain tissue is wavelength dependent, the 715- and 900-nm images reveal spectral differences corresponding to different molecular constituents. ${ }^{16}$ While hemoglobin provides the major contrast at $715 \mathrm{~nm}$, at $900 \mathrm{~nm}$, water and adipose tissue are expected to contribute more to contrast generation.

Comparison of the intensity profiles obtained from three defined anatomical references revealed subtle but interesting differences. Figure $4(\mathrm{~g})$ (I to III) present the intensity profiles extracted from locations denoted in Figs. 4(d) and 4(f) (I, II, and III), respectively. The normalized graphs ( $\mathrm{g}$, left column) correspond to MSOT512 (black) and MSOT256 (blue, dashed). For comparison of the resolving power of MSOT512 versus MSOT256, we fitted Gaussian curves to the normalized intensity profiles (I to III) and computed their relative FWHM. Figure 4(g) (middle and right columns) shows Gaussian fits for the MSOT256 and MSOT512 measurements, respectively. The computed FWHM values are summarized in Table 2 and advocate improved imaging resolution with MSOT512.

\subsection{Hybrid Imaging of Abdominal Organs}

Figure 5 presents a cryosection of the mouse abdomen ex vivo annotated for major anatomical references (a), with corresponding UST and MSOT cross sections obtained in vivo (b and c, respectively) and their coregistered overlay (d). High absorbance of major blood supply routes, such as spinal branch of lumbar arteries and vertebral column with adjacent caudal vena cava along with large parenchymal organs such as left 
(a)

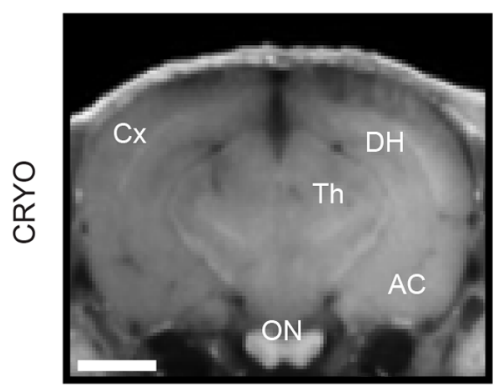

(b)

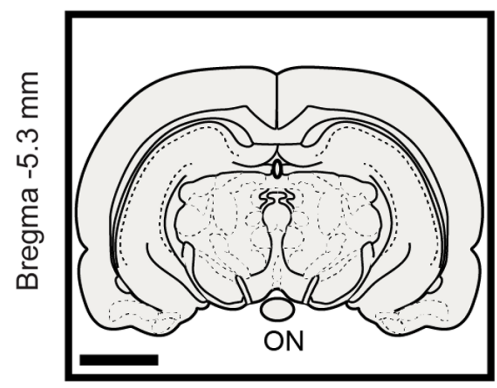

(g)
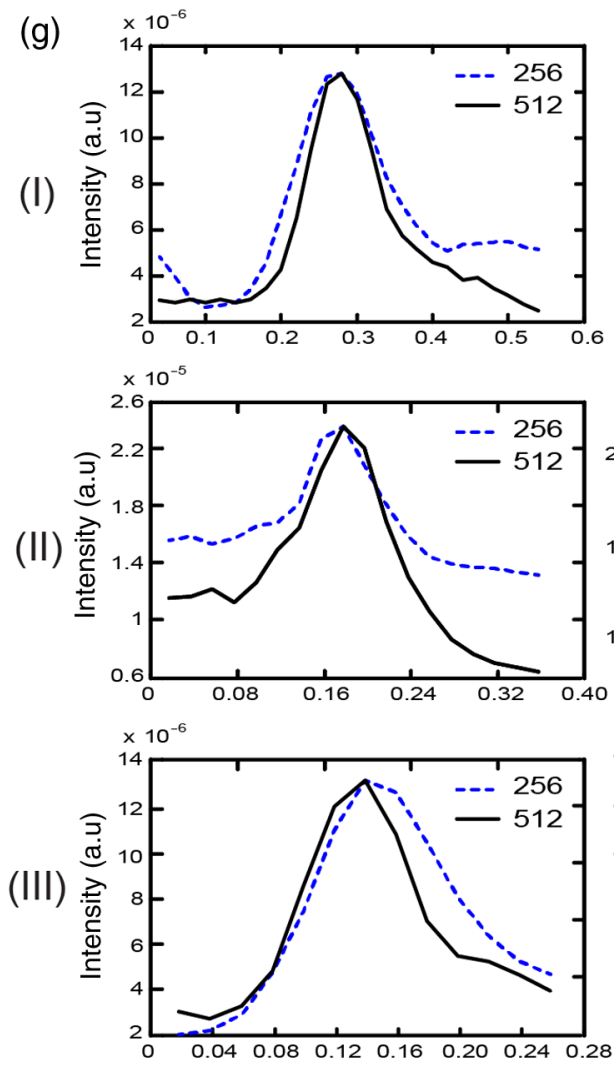

$(\mathrm{mm})$

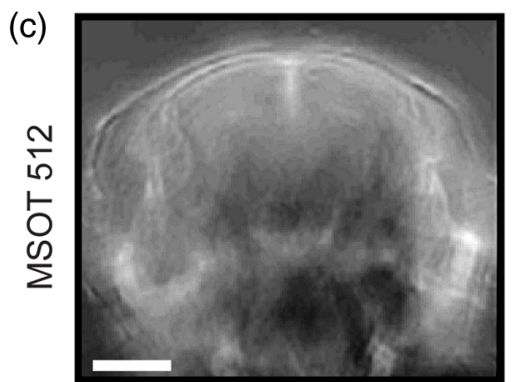

$715 \mathrm{~nm}$

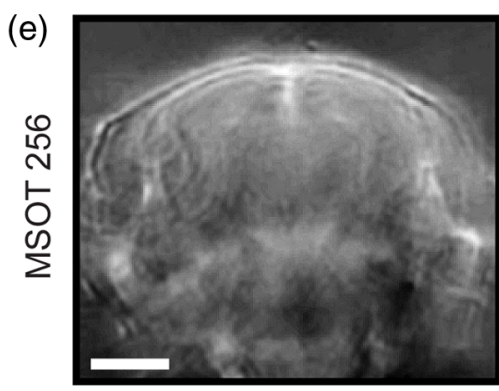

$715 \mathrm{~nm}$
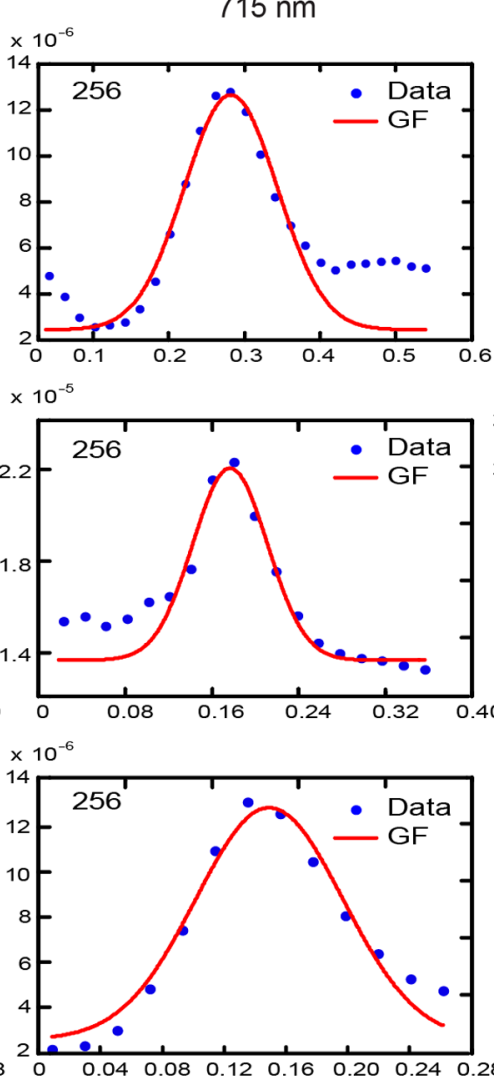

$(\mathrm{mm})$

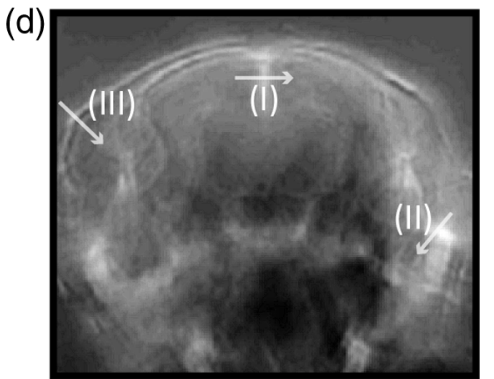

$900 \mathrm{~nm}$

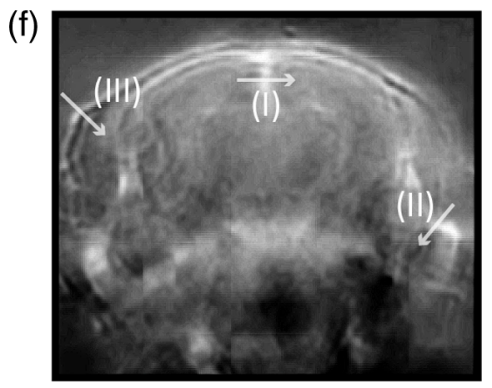

$900 \mathrm{~nm}$
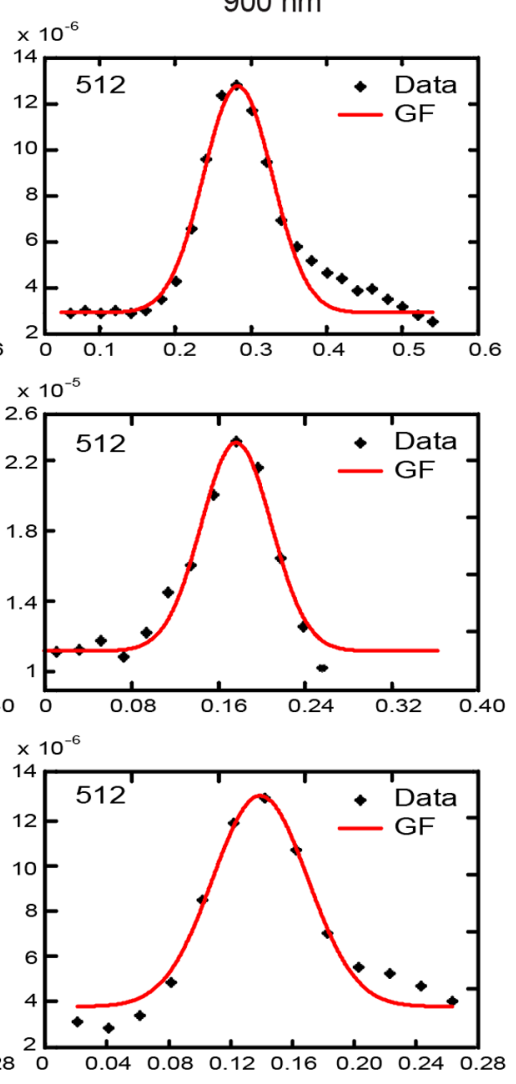

$(\mathrm{mm})$

Fig. 4 Intravital imaging of a mouse brain with MSOT256 and MSOT512: a comparative analysis. (a) Annotated histological cross section (HIST) of a mouse brain with (b) schematic representation of the corresponding anatomical cross section of the brain: $\mathrm{Cx}$, cortex; $\mathrm{DH}$, dorsal hippocampus; Th, thalamus; AC, amygdalar complex; and ON, optic nerve. Scale bars: $2 \mathrm{~mm}$. (c-f) Tomographic images of the mouse brain obtained with MSOT512 (c and d) and MSOT256 (e and f) at $715 \mathrm{~nm}$ (c and e) and $900 \mathrm{~nm}$ ( $\mathrm{d}$ and f). Arrows (I-III) mark approximate locations of intensity profiles extractions. Scale bars: $2.5 \mathrm{~mm}$. (g) Summary of intensity profiles extracted from the coregistered images (d and f). Intensity profiles from MSOT256 (blue, dashed) and MSOT512 (black, solid) are matched at their maxima (left column), fitted with the Gaussian functions (middle and right columns). The FWHM values are presented in Table 2. 
Table 2 Comparative FWHM fits of the intensity profiles of sagittal sinus, maxillary artery, and subcutaneous artery using MSOT256 and MSOT512 (units in $\mathrm{mm}$ ).

\begin{tabular}{lccc}
\hline Scanners & $\begin{array}{c}\text { Sagittal } \\
\text { sinus (I) }\end{array}$ & $\begin{array}{c}\text { Maxillary } \\
\text { artery (II) }\end{array}$ & $\begin{array}{c}\text { Subcutaneous } \\
\text { artery (III) }\end{array}$ \\
\hline MSOT256 & 0.146 & 0.106 & 0.083 \\
MSOT512 & 0.104 & 0.078 & 0.074 \\
Difference (\%) & 28.661 & 32.425 & 10.592 \\
\hline
\end{tabular}

and right kidneys, spleen, the head of the pancreas, and epigastria vessels, is visible on MSOT images. UST images, on the other hand, offer better contrast and visualization of organs at greater depths, revealing kidneys, liver, pancreas, vertebral column with the spinal cord and other structures. Importantly, blood reach formations, such as medium and small vascular elements, and parts of liver hardly distinguishable on UST images are revealed better by MSOT, while collagen and connective tissue rich structures and bones reveal higher contrast at UST. Thus, UST of the abdominal organs unveils rich anatomical data on deeper structures, while MSOT provides complementary features based on optical contrast. The latter is especially important for functional imaging, as it affords intravital assessment of local $\mathrm{sO}_{2}$ and hemoglobin concentration. Figures 5(e) and 5(f) present abdominal cross sections merged with color-coded oxygenation maps computed from MSOT data corresponding to two different breathing phases [air (e) and $100 \% \mathrm{O}_{2}$ (f)]. Similar to brain measurements, assessment of

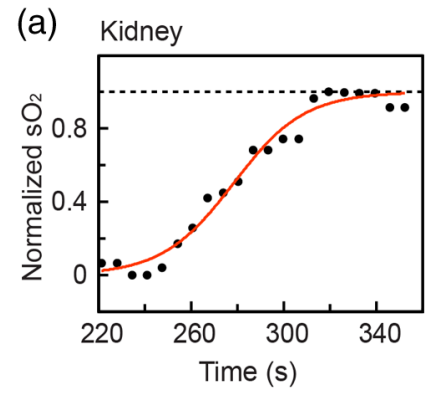

(b) Abdominal blood vessel

Fig. 6 Graphical representation of the kinetics of the $\mathrm{sO}_{2}$ within large blood vessels and parenchyma of abdominal organs measured with MSOT. ( $a$ and $b$ ) Representative $\mathrm{sO}_{2}$ dynamics overtime pulled from ROIs [corresponding to arrows: 1 and 3 , in Fig. 5(c), respectively]. Individual data points overtime are fitted with sigmoid functions with values (tau) defining the slope summarized in Table 1.

the $\mathrm{sO}_{2}$ kinetics of abdominal organs under different breathing conditions in two ROIs (enboxed 1 and 4) revealed clear correspondence of the hemodynamics with the composition of supplied gas, with dynamics of the $\mathrm{sO}_{2}$ changes related to the gas challenges readily visible [Figs. 5(g) and 5(h)]. After baseline recording while breathing air, supply of $\mathrm{CO}_{2}$ causes gradual deoxygenation of the abdominal structures while supply of pure $\mathrm{O}_{2}$ results in rapid increase in tissue and blood oxygenation. Similar to the brain, these processes remain within physiological limits and reversed upon supply of normal breathing air.

To evaluate the kinetics of the hemoglobin gradient changes in abdominal organs, we analyzed the rates of the relative $\mathrm{sO}_{2}$ changes upon $\mathrm{O}_{2}$ challenge. Figure 6 presents the analysis of (a)

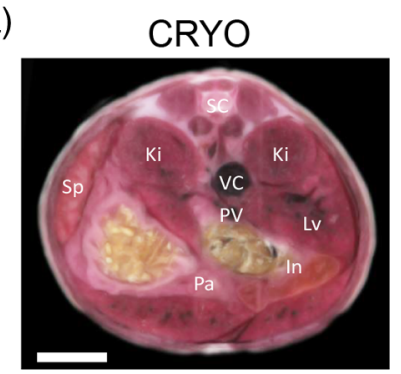

(c)

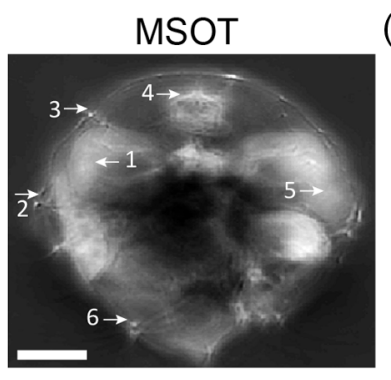

(b)

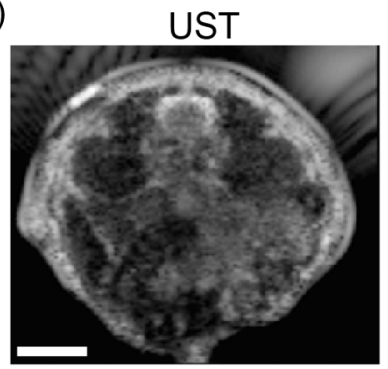

(d)

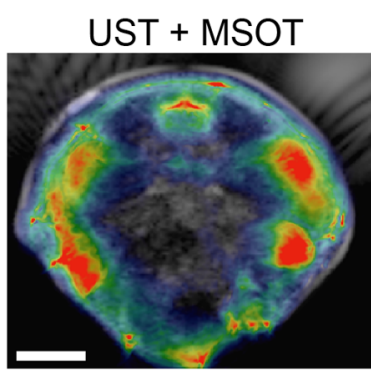

(e)

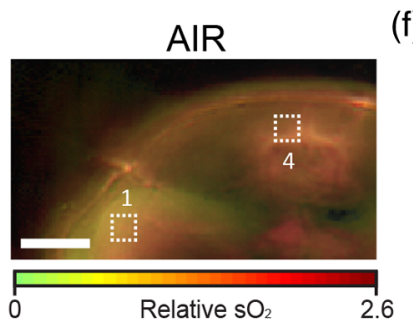

(f)

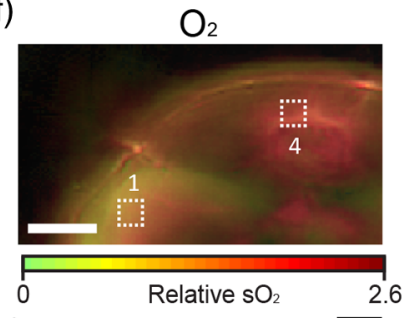

(g)

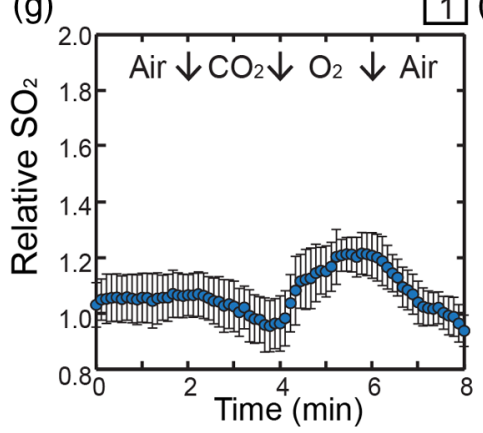

(h)

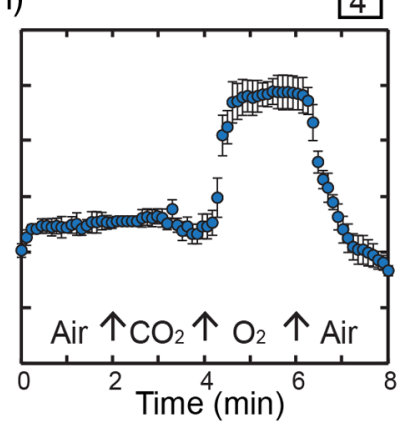

Fig. 5 Hybrid UST and MSOT imaging of abdominal organs. (a) Annotated cross section of a mouse abdomen with UST, MSOT, and overlaid images (b, c, and d, respectively). Annotations: SC, spinal cord; Ki, kidney; Sp, spleen; VC, vena cava; PV, portal vein; Pa, pancreas; Lv, liver; and In, intestine. Scale bars: $3 \mathrm{~mm}$. The regions of interest (1 to 6) for analysis of the $\mathrm{sO}_{2}$ dynamics are highlighted on (c) MSOT image with arrows (summarized in Table 1). 1 and 5, kidneys; 2, 3, and 6, superficial vessels; and 4, spinal branch of lumbar artery. (e and f) Color-coded relative $\mathrm{sO}_{2}$ maps obtained at indicated breathing phases overlaid with anatomical images. ( $g$ and $h$ ) Time courses of changes from respective regions of interests (enboxed, 1 and 4). Blue circles and error bars denote mean relative $\mathrm{sO}_{2}$ and standard deviations of measurements within respective ROls, correspondingly. Experimental conditions are indicated within individual graphs. Scale bars: $2 \mathrm{~mm}$. 
the $\mathrm{sO}_{2}$ kinetics of kidney tissue (a) and an abdominal blood vessel (b), with data obtained from regions marked by arrows 1 and 3 in Fig. 5(c), respectively. Black circles correspond to the normalized data points with red lines showing the fitted sigmoid functions. Parenchymal organs showed slower $\mathrm{sO}_{2}$ rates compared to those of the brain (Table 1), supporting tighter coupling of the brain $\mathrm{sO}_{2}$ with breathing state and overall greater oxygen consumption of neural tissue and its lower tolerance to hypoxia. ${ }^{42,43}$

\section{Discussion}

Hybrid imaging systems offer a highly promising future to correlative biomedical and clinical studies. At present, dual PET-CT implementations are widely applied for combining the intravital molecular imaging with high-resolution structural information. ${ }^{44,45}$ Likewise, hybrid XCT and fluorescence molecular tomography have proven more instructive compared to stand-alone counterparts. ${ }^{46,47}$ In this study, we applied a MSOT-UST system for structural and functional spectral OA and ultrasound imaging and validated its performance on the head and abdominal organs in adult mice in vivo.

The combination of ultrasound and multispectral OA imaging for interrogation of biological specimens has been considered in the past with the use of linear DAs. ${ }^{32,48}$ However, the latter has proven to be unable to produce volumetric OA readouts, with imaging capabilities limited to 2 -D visualization of superficial layers of the specimen. Recently, curved-array MSOT-UST system has been developed and used for imaging the entire mouse in tomographic mode. ${ }^{36}$ This system was not, however, considered for imaging functional processes unfolding within the brain or abdominal organs. The capability of the curved DA to visualize the entire animal at ultrasound diffraction-limited resolution combined with high frame rate measurements of functional dynamics are especially attractive for reconstructing physiological processes in near real-time with their correlation with structural data. In this study, we used curved-array MSOT-UST system for dual structural and functional imaging in vivo to characterize the performance of both modalities and highlight advantages and limitations of using such a hybrid system for experimental and preclinical research. As expected, hybrid images exhibited complementary features. Indeed, on the head scans, UST clearly revealed the boundaries between various tissue types, with skull reflecting most of the ultrasound waves and, thus, appearing as a high contrast; the brain in these images was hardly visible. Thus, the structural US images of the brain have limited diagnostic and exploratory value, as expected. Nevertheless, US modality provides numerous benefits in brain imaging applications. Indeed, MSOT reconstruction suffers from acoustic inhomogeneity of skull that can be corrected to some extent by incorporating structural information provided by US into OA image reconstruction process. It could also help delineating brain region in MSOT images. MSOT images, in turn, revealed hemoglobin gradients with strongest signals corresponding to the compartments with high blood content such as large vessels, inner parenchymal organs, and the brain. The latter, however, comes with considerable deterioration of the image quality with increase of the imaging depth. Due to high optical absorption of the hemoglobin, MSOT readouts contain important functional information on hemodynamics, which is of direct relevance to the level of tissue oxygenation and metabolism rate. This capability of MSOT is particularly attractive in light of growing interest in direct measurements of the oxygen consumption and hemoglobin gradients in vivo. With further improvements and corrections for aberrations and signal loss within highly scattering tissues, MSOT could provide powerful means for simultaneous structural and multispectral functional imaging with better correlation of the UST data. The latter should facilitate also accurate representation of molecular data in the context of anatomical readout, including the biodistribution of reporters and contrast agents as well as changes in the hemodynamics and metabolic activity in various tissues and organs. It is worth noting that unlike the brain, US imaging of the abdomen reveals more clearly structural features and contours of inner organs and is capable of visualizing tissue interfaces at greater depths. In turn, MSOT exhibits worse performance in terms of structural imaging due to attenuation of light with depth and provides complimentary optical contrasts based on the hemoglobin concentrations and blood oxygenation with the ability to track the $\mathrm{sO}_{2}$ dynamics. Such correlative arrangement greatly enriches the content of obtained anatomical and functional data and should facilitate better characterization and understanding of a wide range of biological processes and functions. Importantly, MSOT measurements of the hemodynamics relate meaningfully with applied gas challenges, with physiological values matching closely those reported previously. ${ }^{49}$ The onset rates of $\mathrm{sO}_{2}$ changes defined here are also within the expected kinetic ranges. ${ }^{50}$ Combined with exogenous labels and reporters, ${ }^{18}$ further advancements of hybrid MSOT-UST imaging should facilitate the interrogation processes with direct relevance to pharmacokinetics, biodiagnostics, and pathophysiological studies. ${ }^{28,51-54}$ With regard to MSOT imaging quality, it should be noted that the implemented ultrasound array with 512 transducers moderately improves MSOT resolution as compared to its predecessor with 256 element detectors. Better resolution of the selected profiles shown here agrees with the experimental phantom results ${ }^{34,36}$ as well as theoretical predictions ${ }^{55}$ reported previously.

At this stage, major technical challenges remain ahead that need to be addressed. One area that requires attention is motion artifacts related with the breathing of experimental mice, which due to small animal size, cause incongruity between corresponding MSOT and UST images. A semiautomatic approach for relating MSOT and UST data can be taken to partially overcome this problem, with registration performed based on carefully selected reference points. The main difficulty with this approach stems from the fact that differences in contrasts and penetration between two modalities make the choice of structural references nontrivial. Further research is currently on the way to address these challenges and optimize the signal propagation with more effective data collection. Notwithstanding, described here hybrid system holds great potential for gaining new insights into numerous questions, which necessitate concurrent acquisition with correlation of structural, functional, and molecular data.

To conclude, as a modality with numerous applications in the biological imaging, MSOT is increasingly used for neuroimaging. ${ }^{56}$ The multispectral capability that affords its molecular specificity and ability to differentiate numerous endogenous and exogenous absorbers make it a valuable tool for brain imaging with potential applications ranging from high-resolution structural mesoscopy to imaging of functional processes involving changes of hemoglobin and neural dynamics, vascular dynamics, metabolism (i.e., oxygen consumption rate), and biodistribution of contrasts and drugs. Along with 
pertinence for basic animal research, MSOT has also growing implications for preclinical studies in animal models and potentially in humans. Indeed, reports from animal model of Parkinson's disease, Alzheimer's disease, epilepsy, and stroke have shown the feasibility and usefulness of OA imaging, yielding new insights into the disease mechanisms. With further optimizations, OA tools are likely to be used in the near future also in human studies.

\section{Acknowledgments}

This work was supported by G.W. Leibnitz Price DFG (2013) to V.N. and PRISAR grant (EU) to V.N.

\section{References}

1. R. Salzer, Biomedical Imaging: Principles and Applications, John Wiley \& Sons, Incorporated, Hoboken, New Jersey (2008).

2. F. Kiessling, J. Pichler, and P. Hauff, Small Animal Imaging, Springer, Heidelberg (2011).

3. E. Mark et al., "In vivo validation of the bold mechanism: a review of signal changes in gradient echo functional MRI in the presence of flow," Int. J. Imaging Syst. Technol. 6(2-3), 153-163 (1995).

4. E. R. Sowell et al., "Development of cortical and subcortical brain structures in childhood and adolescence: a structural MRI study," Dev. Med. Child Neurol. 44(1), 4-16 (2002).

5. T. L. Szabo, Diagnostic Ultrasound Imaging: Inside Out, Academic Press, Amsterdam, Boston (2004).

6. K. Kubota, "From tumor biology to clinical PET: a review of positron emission tomography (PET) in oncology," Ann. Nucl. Med. 15(6), 471486 (2001).

7. H. T. Benamer et al., "Accurate differentiation of Parkinsonism and essential tremor using visual assessment of [123I]-FP-CIT SPECT imaging: The [123I]-FP-CIT study group," Mov. Disorders 15(3), 503-510 (2000).

8. W. A. Kalender, "X-ray computed tomography," Phys. Med. Biol. 51(13), R29 (2006).

9. V. Andresen et al., "Infrared multiphoton microscopy: subcellularresolved deep tissue imaging," Curr. Opin. Biotechnol. 20(1), 54-62 (2009).

10. J. A. Izatt et al., "Micrometer-scale resolution imaging of the anterior eye in vivo with optical coherence tomography," Arch. Ophthalmol. 112(12), 1584-1589 (1994).

11. V. Ntziachristos, "Going deeper than microscopy: the optical imaging frontier in biology," Nat. Methods 7(8), 603-614 (2010).

12. P. Beard, "Biomedical photoacoustic imaging," Interface Focus 1(4), 602-631 (2011).

13. V. Ntziachristos et al., "Looking and listening to light: the evolution of whole-body photonic imaging," Nat. Biotechnol. 23(3), 313-320 (2005).

14. L. V. Wang and S. Hu, "Photoacoustic tomography: in vivo imaging from organelles to organs," Science 335(6075), 1458-1462 (2012).

15. A. G. Bell, "On the production and reproduction of sound by light," Am. J. Sci. 20, 305-324 (1880).

16. V. Ntziachristos and D. Razansky, "Molecular imaging by means of multispectral optoacoustic tomography (MSOT)," Chem. Rev. 110(5), 2783-2794 (2010).

17. A. Petschke and P. J. La Riviere, "Comparison of intensity-modulated continuous-wave lasers with a chirped modulation frequency to pulsed lasers for photoacoustic imaging applications," Biomed. Opt. Express 1(4), 1188-1195 (2010).

18. A. Taruttis and V. Ntziachristos, "Advances in real-time multispectral optoacoustic imaging and its applications," Nat. Photonics 9, 219-227 (2015).

19. S. Tzoumas et al., "Unmixing molecular agents from absorbing tissue in multispectral optoacoustic tomography," IEEE Trans. Med. Imaging 33(1), 48-60 (2014).

20. A. A. Oraevsky, "Contrast agents for optoacoustic imaging: design and biomedical applications," Photoacoustics 3(1), 1-2 (2015).

21. A. Liopo, R. Su, and A. A. Oraevsky, "Melanin nanoparticles as a novel contrast agent for optoacoustic tomography," Photoacoustics 3(1), 3543 (2015).
22. D. Razansky et al., "Multispectral opto-acoustic tomography of deepseated fluorescent proteins in vivo," Nat. Photonics 3(7), 412-417 (2009).

23. J. A. Copland et al., "Bioconjugated gold nanoparticles as a molecular based contrast agent: implications for imaging of deep tumors using optoacoustic tomography," Mol. Imaging Biol. 6(5), 341-349 (2004).

24. A. Taruttis, G. M. van Dam, and V. Ntziachristos, "Mesoscopic and macroscopic optoacoustic imaging of cancer,' Cancer Res. 75(8), 1548-1559 (2015).

25. X. Wang et al., "Noninvasive laser-induced photoacoustic tomography for structural and functional in vivo imaging of the brain," Nat. Biotechnol. 21(7), 803-806 (2003).

26. S. Hu and L. V. Wang, "Neurovascular photoacoustic tomography," Front Neuroenerg. 2, 10 (2010).

27. A. Buehler et al., "High resolution tumor targeting in living mice by means of multispectral optoacoustic tomography," EJNMMI Res. 2, 14 (2012).

28. A. Taruttis et al., "Real-time imaging of cardiovascular dynamics and circulating gold nanorods with multispectral optoacoustic tomography," Opt. Express 18(19), 19592-19602 (2010).

29. G. J. Tserevelakis et al., "Hybrid multiphoton and optoacoustic microscope," Opt. Lett. 39(7), 1819-1822 (2014).

30. J. J. Niederhauser et al., "Combined ultrasound and optoacoustic system for real-time high-contrast vascular imaging in vivo," IEEE Trans. Med. Imaging 24(4), 436-440 (2005).

31. M. Lakshman and A. Needles, "Screening and quantification of the tumor microenvironment with micro-ultrasound and photoacoustic imaging," Nat. Methods 12(4), iii-v (2015).

32. S. A. Ermilov et al., "Development of laser optoacoustic and ultrasonic imaging system for breast cancer utilizing handheld array probes," Proc. SPIE 7177, 717703 (2009).

33. C. Kim et al., "Deeply penetrating in vivo photoacoustic imaging using a clinical ultrasound array system," Biomed. Opt. Express 1(1), 278-284 (2010).

34. A. Dima, N. C. Burton, and V. Ntziachristos, "Multispectral optoacoustic tomography at 64, 128, and 256 channels," J. Biomed. Opt. 19(3), 036021 (2014).

35. J. Xia et al., "Enhancement of photoacoustic tomography by ultrasonic computed tomography based on optical excitation of elements of a full-ring transducer array," Opt. Lett. 38(16), 3140-3143 (2013).

36. E. Mercep et al., "Whole-body live mouse imaging by hybrid reflectionmode ultrasound and optoacoustic tomography," Opt. Lett. 40(20), 4643-4646 (2015).

37. E. Mercep et al., "Hybrid optoacoustic tomography and pulse-echo ultrasonography using concave arrays," IEEE Trans. Ultrason. Ferroelectr. Freq. Control 62(9), 1651-1661 (2015).

38. A. Buehler et al., "Video rate optoacoustic tomography of mouse kidney perfusion," Opt. Lett. 35(14), 2475-2477 (2010).

39. T. Iwaki, A Color Atlas of Sectional Anatomy of the Mouse, Braintree Scientific Inc., Tokyo, Japan (2001).

40. D. Lu et al., "Efficient non-negative constrained model-based inversion in optoacoustic tomography," Phys. Med. Biol. 60(17), 6733-6750 (2015).

41. G. Paxinos and C. Watson, The Rat Brain in Stereotaxic Coordinates, 6th ed., Academic Press/Elsevier, Amsterdam, Boston (2007).

42. V. Jain, M. C. Langham, and F. W. Wehrli, "MRI estimation of global brain oxygen consumption rate," J. Cereb. Blood Flow Metab. 30(9), 1598-1607 (2010).

43. F. Xu, Y. Ge, and H. Lu, "Noninvasive quantification of whole-brain cerebral metabolic rate of oxygen (CMRO2) by MRI," Magn. Reson. Med. 62(1), 141-148 (2009).

44. P. E. Kinahan et al., "Attenuation correction for a combined 3D PET/CT scanner," Med. Phys. 25(10), 2046-2053 (1998).

45. T. Beyer et al., "A combined PET/CT scanner for clinical oncology," J. Nucl. Med. 41(8), 1369-1379 (2000).

46. A. Ale et al., "FMT-XCT: in vivo animal studies with hybrid fluorescence molecular tomography-x-ray computed tomography," Nat. Methods 9(6), 615-620 (2012).

47. A. Ale et al., "Fluorescence background subtraction technique for hybrid fluorescence molecular tomography/x-ray computed tomography imaging of a mouse model of early stage lung cancer," J. Biomed. Opt. 18(5), 056006 (2013). 
48. K. Daoudi et al., "Handheld probe integrating laser diode and ultrasound transducer array for ultrasound/photoacoustic dual modality imaging," Opt. Express 22(21), 26365-26374 (2014).

49. N. C. Burton et al., "Multispectral opto-acoustic tomography (MSOT) of the brain and glioblastoma characterization," NeuroImage $\mathbf{6 5}, 522$ 528 (2013).

50. S. Gottschalk et al., "Noninvasive real-time visualization of multiple cerebral hemodynamic parameters in whole mouse brains using fivedimensional optoacoustic tomography," J. Cereb. Blood Flow Metab. 35(4), 531-535 (2015).

51. M. L. Li et al., "Simultaneous molecular and hypoxia imaging of brain tumors in vivo using spectroscopic photoacoustic tomography," Proc. IEEE 96(3), 481-489 (2008).

52. L. D. Liao et al., "Transcranial imaging of functional cerebral hemodynamic changes in single blood vessels using in vivo photoacoustic microscopy," J. Cereb. Blood Flow Metab. 32(6), 938-951 (2012).
53. A. Taruttis et al., "Multispectral optoacoustic tomography of myocardial infarction," Photoacoustics 1(1), 3-8 (2013).

54. A. Taruttis et al., "Fast multispectral optoacoustic tomography (MSOT) for dynamic imaging of pharmacokinetics and biodistribution in multiple organs," PLoS One 7(1), e30491 (2012).

55. M. Xu and L. V. Wang, "Analytic explanation of spatial resolution related to bandwidth and detector aperture size in thermoacoustic or photoacoustic reconstruction," Phys. Rev. E 67(5), 056605 (2003).

56. J. Yao and L. V. Wang, "Photoacoustic brain imaging: from microscopic to macroscopic scales," Neurophotonics 1(1), 011003 (2014).

Biographies for the authors are not available. 